\title{
Arachidonic acid supplementation during gestational, lactational and post-weaning periods prevents retinal degeneration induced in a rodent model
}

\author{
Katsuhiko Yoshizawa ${ }^{1 *}$, Tomo Sasaki ${ }^{1,2}$, Maki Kuro ${ }^{3}$, Norihisa Uehara ${ }^{1}$, Hideho Takada ${ }^{4}$, \\ Akiko Harauma $^{5}$, Naoki Ohara ${ }^{6}$, Toru Moriguchi ${ }^{5}$ and Airo Tsubura ${ }^{1}$ \\ ${ }^{1}$ Department of Pathology II, Kansai Medical University, 10-15 Fumizono, Moriguchi, Osaka 570-8506, Japan \\ ${ }^{2}$ Kyoto RED Center, Marubo Company Limited, Kyoto 600-8815, Japan \\ ${ }^{3}$ Department of Ophthalmology, Kansai Medical University Takii Hospital, Moriguchi, Osaka 570-8507, Japan \\ ${ }^{4}$ Clinical Department of Surgery, Kansai Medical University Takii Hospital, Moriguchi, Osaka 570-8506, Japan \\ ${ }^{5}$ Laboratory of Food and Nutritional Science, Life and Environmental Science, Azabu University, Sagamihara, Kanagawa \\ 252-5201, Japan \\ ${ }^{6}$ School of Pharmacy, Kinjo Gakuin University, Nagoya, Aichi 463-8521, Japan
}

(Submitted 8 March 2012 - Final revision received 23 May 2012 - Accepted 28 June 2012 - First published online 6 August 2012)

\begin{abstract}
Fatty acids and their derivatives play a role in the response to retinal injury. The effects of dietary arachidonic acid (AA) supplementation on $N$-methyl- $N$-nitrosourea (MNU)-induced retinal degeneration was investigated in young Lewis rats during the gestational, lactational and post-weaning periods. Dams were fed $0 \cdot 1,0 \cdot 5$ or $2 \cdot 0 \%$ AA diets or a basal $(<0 \cdot 01 \% \mathrm{AA})$ diet. On postnatal day 21 (at weaning), male pups received a single intraperitoneal injection of $50 \mathrm{mg} \mathrm{MNU} / \mathrm{kg}$ or vehicle, and were fed the same diet as their mother for $7 \mathrm{~d}$. Retinal apoptosis was analysed by the terminal deoxynucleotidyl transferase-mediated dUTP digoxigenin nick-end labelling (TUNEL) assay $24 \mathrm{~h}$ after the MNU treatment, and retinal morphology was examined $7 \mathrm{~d}$ post-MNU. Histologically, all rats that received MNU and were fed the basal and $0.1 \%$ AA diets developed retinal degeneration characterised by the loss of photoreceptor cells (disappearance of the outer nuclear layer and the photoreceptor layer) in the central retina. The 0.5 and $2.0 \%$ AA diets rescued rats from retinal damage. Morphometrically, in parallel with the AA dose $(0.5$ and $2.0 \% \mathrm{AA})$, the photoreceptor ratio significantly increased and the retinal damage ratio decreased in the central retina, compared with the corresponding ratios in basal diet-fed rats. In parallel with the increase in serum and retinal AA levels and the AA:DHA ratio, the apoptotic index in the central retina was dose-dependently decreased in rats fed the 0.5 and $2.0 \%$ AA diets. In conclusion, an AA-rich diet during the gestation, lactation and post-weaning periods rescued young Lewis rats from MNU-induced retinal degeneration via the inhibition of photoreceptor apoptosis. Therefore, an AA-enriched diet in the prenatal and postnatal periods may be an important strategy to suppress the degree of photoreceptor injury in humans.
\end{abstract}

Key words: Arachidonic acid: $\mathrm{N}$-methyl- $\mathrm{N}$-nitrosourea: Retinal degeneration, Retinitis pigmentosa

Arachidonic acid (AA) is a PUFA present in the phospholipids of cell membranes, and it is particularly abundant in the retina and brain $^{(1,2)}$. AA in the human body comes from dietary sources such as egg yolk ${ }^{(2)}$, or it is synthesised from linoleic $\operatorname{acid}^{(3)}$. Neurological health requires sufficient levels of DHA and $\mathrm{AA}^{(4)}$. Early infancy may be a critical time when visual development and brain development are susceptible to the effects of inadequate stores or a deficient intake of essential fatty acids, DHA and $\mathrm{AA}^{(5)}$. AA drives postnatal neurogenesis and elicits a beneficial effect on prepulse inhibition in Pax6 knockout mice characterised by impaired postnatal neurogenesis ${ }^{(6)}$. Several randomised clinical trials of supplemental DHA and AA have been conducted in full-term infants, and infants who received the supplementation had better visual-evoked potential acuity when compared with the control groups ${ }^{(2,7)}$. Recently, nutrient-based preventive treatments for development and progression on human retinal diseases have been focused, and large-scale clinical studies, such as the Age-Related Eye Disease Study, have been conducted ${ }^{(8)}$.

Inherited night blindness is a fairly widespread disease in humans, and it affects approximately one in 5000 individuals worldwide. A common form of inherited blindness is retinitis

Abbreviations: AA, arachidonic acid; MNU, $N$-methyl- $N$-nitrosourea; RP, retinitis pigmentosa; TUNEL, terminal deoxynucleotidyl transferase-mediated dUTP digoxigenin nick-end labelling.

*Corresponding author: Dr K. Yoshizawa, fax +8166992 5023, email yoshizak@takii.kmu.ac.jp 
pigmentosa (RP), which is a degenerative pigmentary retinopathy that is non-inflammatory, bilateral and progressive ${ }^{(9,10)}$. $\mathrm{RP}$ is a heterogeneous group of inherited retinal disorders caused by more than 160 different mutations of genes encoding proteins with remarkably diverse functions that are known to cause photoreceptor degeneration ${ }^{(11)}$. In many RP cases, clinical symptoms typically start in the early teenage years and severe visual impairment occurs by ages $40-50$ years $^{(12)}$. Patients with Usher syndrome, roughly one-sixth of patients with RP, begin to lose their vision in their first decade of life ${ }^{(13)}$. This type can be diagnosed before birth by using genetic methods and/or a pedigree diagram. In these cases, the initiation of therapy before birth may prevent or delay retinal damage in young people with RP.

Animal models of retinal degeneration are important for elucidating the mechanisms of human $\mathrm{RP}^{(14,15)}$ and exploring potential treatments ${ }^{(10,16,17)}$. Mutant mice used as models for $\mathrm{RP}$ include mice carrying the rodless retina or retinal degeneration $(r d)$ gene, or the retinal degeneration slow $(r d s)$ gene; these mice develop photoreceptor cell death caused by apoptosis $^{(15)}$. In addition to inherited RP models, $N$-methyl- $N$-nitrosourea (MNU), an alkylating agent that targets photoreceptor cells, rapidly induces retinal damage via apoptosis in several animal species ${ }^{(16,17)}$. MNU-induced retinal damage is due to selective 7-methyldeoxyguanosine DNA adduct formation in photoreceptor cell nuclei followed by photoreceptor cell death via an apoptotic mechanism ${ }^{(18)}$. The MNU-induced apoptosis cascade involves the up-regulation of Bax protein, the down-modulation of $\mathrm{Bcl}-2$ protein and the activation of caspase-3, -6 and $-8^{(18)}$. Within $7 \mathrm{~d}$ after an adequate dose of MNU is administered, active signs of photoreceptor degeneration are distinct due to photoreceptor cell loss, and the inner nuclear layer is either in direct contact with the choroid or is separated from it by a few layers of cells. This model mimics the cell death process in human RP and can be used for studies of therapeutic intervention. The goal of the present study is to elucidate the effect of prenatal and postnatal dietary AA on MNU-induced retinal degeneration in young Lewis rats.

\section{Experimental materials}

\section{Animal procedures}

The protocol and all animal procedures used in the present experiment were approved by the Animal Care and Use Committee of Kansai Medical University and were in accordance with the guidelines for animal experimentation at the Kansai Medical University. A total of thirty-one male and female $\mathrm{SPF} / \mathrm{VAF}$ rats (LEW/CrlCrlj) at 10-12 weeks of age were purchased from Charles River Japan. In our previous study, we confirmed that MNU induces retinal degeneration in both male and female rats, without the sex difference in incidence and severity. Lewis inbred rats have a similar sensitivity of retinal damage to the other strain. Therefore, we selected male Lewis rats in the present study. Rats were maintained in specific pathogen-free conditions and received free access to water and CE-2-modified diets containing different doses of AA. The animals were housed in plastic cages with paper-chip bedding (Paper Clean; SLC) in an air-conditioned room at $22 \pm 2^{\circ} \mathrm{C}$ and $60 \pm 10 \%$ relative humidity with a $12 \mathrm{~h}$ light-dark cycle. Illumination intensity in the cages was less than 60 lux. In each dietary group, each male was mated with six to nine females. Offspring were culled to a maximum of ten per dam, and the dams were maintained on their respective diets during the $21 \mathrm{~d}$ lactation period and a post-weaning period of up to $7 \mathrm{~d}$. At the age of $21 \mathrm{~d}$, male pups were selected and used in the present study (Fig. 1) In each dietary group, necropsy was done simultaneously for five vehicle-treated rats and four to eight MNU-treated rats

\section{Chemical and dose formulation}

MNU was obtained from Sigma-Aldrich and was kept at $-80^{\circ} \mathrm{C}$ in the dark. The MNU solution was dissolved in physiological saline containing $0.05 \%$ acetic acid just before use. MNU $(50 \mathrm{mg} / \mathrm{kg}$ ) or vehicle (physiological saline containing $0.05 \%$ acetic acid) was administered once by intraperitoneal injection $^{(18,19)}$.

\section{Arachidonic acid-supplemented diet}

AA (CABIO, purity of $40.4 \%$ by analysis) was purchased from Cargill Alking Bioengineering (Wuhan) Company Limited and purified by T. M. for use. Diets with three concentrations of AA $(0.1,0.5$ and $2.0 \%(\mathrm{w} / \mathrm{w}))$ were semi-purified based on the modified CE-2 formulation (CLEA Japan, Inc.). The basal diet consisted of modified CE-2. The different concentrations of AA were added to prepare each experimental diet. GC analyses of the fatty acid composition of the diets are shown in Table $1^{(20)}$. The total fatty acid volumes were $86.75,131.96$, 126.70 and $126.63 \mu \mathrm{g} / \mathrm{mg}$ diet for the basal diet $(<0.01 \%$ (w/w) AA), $0 \cdot 1,0.5$ and $2.0 \%$ AA diet, respectively. These diets were exposed to $\gamma$-rays (30 Gy), and formulated by CLEA Japan, Inc. The diets were stored at $4^{\circ} \mathrm{C}$ to prevent lipid oxidation before use.

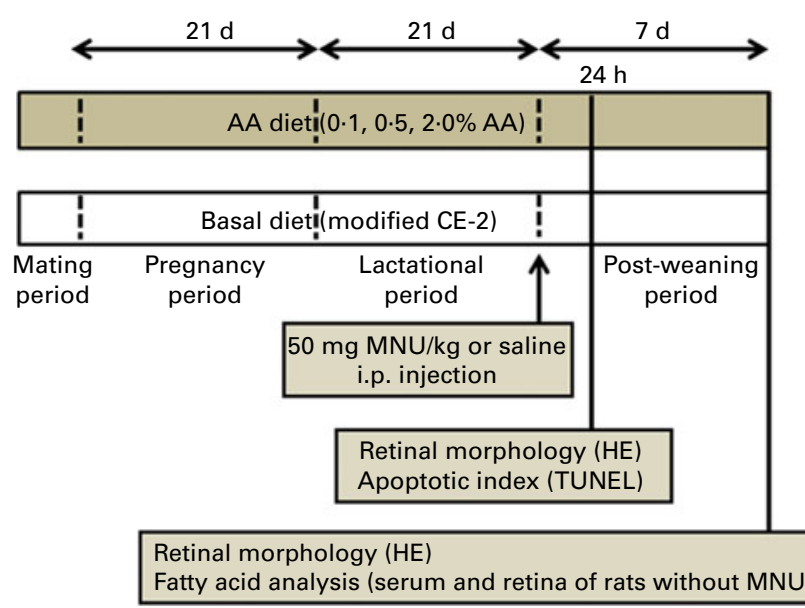

Fig. 1. Schema of the experimental protocol. AA, arachidonic acid; i.p., intraperitoneal; HE, haematoxylin and eosin; TUNEL, terminal deoxynucleotidyl transferase-mediated dUTP digoxigenin nick-end labelling; MNU, $\mathrm{N}$-methyl$N$-nitrosourea. 
Table 1. Fatty acid composition of the diets

\begin{tabular}{|c|c|c|c|c|}
\hline $\begin{array}{l}\text { Fatty acid component } \\
\text { (wt\%) }\end{array}$ & Basal & $0.1 \% \mathrm{AA}$ & $0.5 \%$ AA & $2.0 \% \mathrm{AA}$ \\
\hline$\Sigma$ Saturated & 23.06 & $24 \cdot 19$ & $24 \cdot 22$ & 24.45 \\
\hline ¿Monounsaturated & 34.55 & $37 \cdot 35$ & 34.93 & 24.41 \\
\hline$\Sigma n-6$ & $31 \cdot 82$ & $29 \cdot 21$ & $31 \cdot 68$ & $42 \cdot 26$ \\
\hline$\sum n-3$ & 5.53 & 4.87 & 4.73 & 3.86 \\
\hline$n-3: n-6$ & $0 \cdot 17$ & $0 \cdot 17$ & 0.15 & 0.09 \\
\hline AA:DHA & 0.14 & $2 \cdot 15$ & $8 \cdot 40$ & 32.45 \\
\hline $18: 2 n-6$ & 31.58 & $27 \cdot 88$ & $26 \cdot 91$ & $23 \cdot 73$ \\
\hline $18: 3 n-6$ & 0.00 & 0.07 & 0.22 & 0.85 \\
\hline $20: 2 n-6$ & 0.15 & $0 \cdot 17$ & 0.19 & 0.27 \\
\hline $20: 3 n-6$ & 0.00 & $0 \cdot 10$ & 0.36 & 1.40 \\
\hline $20: 4 n-6$ & $0 \cdot 10$ & 0.99 & 3.95 & $15 \cdot 90$ \\
\hline $22: 2 n-6$ & ND & ND & ND & ND \\
\hline $22: 4 n-6$ & ND & ND & 0.04 & 0.12 \\
\hline $22: 5 n-6$ & ND & ND & ND & ND \\
\hline $18: 3 n-3$ & 3.51 & 3.48 & 3.31 & $2 \cdot 29$ \\
\hline $20: 3 n-3$ & 0.00 & 0.01 & 0.02 & 0.02 \\
\hline $20: 5 n-3$ & 1.07 & 0.75 & 0.79 & 0.91 \\
\hline $22: 5 n-3$ & 0.23 & $0 \cdot 16$ & 0.14 & $0 \cdot 15$ \\
\hline $22: 6 n-3$ & 0.73 & 0.46 & 0.47 & 0.49 \\
\hline Total fatty acid $(\mu \mathrm{g} / \mathrm{mg})$ & $86 \cdot 75$ & 131.96 & $126 \cdot 70$ & $126 \cdot 63$ \\
\hline AA (\% of total fatty acid) & 0.12 & 0.61 & $3 \cdot 12$ & $12 \cdot 56$ \\
\hline
\end{tabular}

$\mathrm{AA}$, arachidonic acid; ND, not determined.

\section{Experimental procedures}

Male Lewis rats were exposed to the basal diet or an experimental diet $(0 \cdot 1,0 \cdot 5$ and $2 \cdot 0 \% \mathrm{AA})$ from fertilisation to killing. At $21 \mathrm{~d}$ of age, these rats received an intraperitoneal injection of vehicle (physiological saline) or $50 \mathrm{mg} \mathrm{MNU/kg} \mathrm{(Fig.} \mathrm{1).} \mathrm{At} 24 \mathrm{~h}$ (five rats in each dietary group) or $7 \mathrm{~d}$ (four to eight rats in each dietary group) after the MNU treatment, rats were anaesthetised with isoflurane (Forane ${ }^{\circledR}$; Abbot Japan) and killed by exsanguination from aortic transection. During the experiment, all pups were observed daily for clinical signs of toxicity and were weighed at the time of MNU treatment and on the day of killing. Both eyes were quickly removed at the time of killing, and complete necropsies were conducted on all animals. Food consumption and the body weight of the dams were measured once per week to estimate the actual dosage of AA during the mating, pregnancy and lactation periods.

\section{Fatty acid analysis of retina and serum}

For the analysis, retinas and serum samples were collected from each of the rats killed $7 \mathrm{~d}$ after injection of the vehicle. Retinas and blood samples were stored at $-80^{\circ} \mathrm{C}$ before lipid extraction and the measurement of fatty acid composition. The fatty acid composition was measured as described previously with minor modifications ${ }^{(21,22)}$. Tissue samples were thawed, weighed and homogenised in methanol-hexane and methylated in acetyl chloride. Varying amounts of internal standards, methyl docosatrienoate (22:3n-3 for retina) and methyl tricosanoic (23:0 for serum), were added to each sample to compensate for differences in tissue weight and lipid concentration. As an aid in preventing lipid oxidation during the procedures, butylated hydroxytoluene was added to methanol. The hexane extracts were concentrated into microvials for GC injection. Fatty acid methyl esters were analysed with an Agilent
7890A GC (Agilent Technologies) equipped with a split injector, an Agilent 7693A ALS automatic liquid sampler and an flame ionisation detector. The instrument was controlled and data were collected using Agilent ChemStation (Rev.B.04.01.SP1; Agilent Technologies).

\section{Tissue fixation and processing}

From the saline-treated rats that were fed each diet, one eye was fixed overnight in methacarn. From the MNU-treated rats, one eye was fixed overnight in $10 \%$ neutral buffered formalin, and the other eye was fixed overnight in methacarn. Subsequently, the eyes were embedded in paraffin, sectioned at a thickness of $4 \mu \mathrm{m}$, and stained with haematoxylin and eosin. Ocular sections were cut along a line parallel to the optic axis and nerve (including the ora serrata).

\section{Morphometric analysis of retinal thickness, photoreceptor cell ratio and retinal damage ratio}

Haematoxylin and eosin-stained sections and terminal deoxynucleotidyl transferase-mediated dUTP digoxigenin nick-end labelling (TUNEL)-stained sections of the retina were scanned with a high-resolution digital slide scanner (NanoZoomer $2 \cdot 0$ Digital Pathology; Hamamatsu Photonics) to prepare digital images. The image files were opened in colour mode using NDP.view software (Hamamatsu Photonics). The total retinal thickness (from the internal limiting membrane to the pigment epithelium), inner retinal thickness (from the internal limiting membrane to the outer plexiform layer) and outer retinal thickness (from the outer nuclear layer to the pigment epithelial cell layer) were individually measured from methacarn-fixed haematoxylin and eosin-stained slides by using NDP.view, as described previously ${ }^{(19,23)}$. The measurements were conducted at the central retina (approximately $400 \mu \mathrm{m}$ from the optic nerve) and peripheral retina (approximately $400 \mu \mathrm{m}$ from both sides of the ciliary bodies). To further evaluate the photoreceptor cell loss, the photoreceptor ratio was calculated as:

$$
\begin{aligned}
\text { Photoreceptor ratio }= & (\text { outer retinal thickness } / \\
& \text { total retinal thickness }) \times 100 .
\end{aligned}
$$

To determine the area of retinal damage, the entire length of the retina and the length of the damaged area in haematoxylin and eosin preparations were measured. Damaged retina was designated as the presence of less than four rows of photoreceptor nuclei in the outer nuclear layer ${ }^{(19,23)}$, and the retinal damage ratio was calculated as:

$$
\begin{aligned}
\text { Retinal damage ratio }= & \text { (length of damaged retina } / \\
& \text { whole retinal length }) \times 100 .
\end{aligned}
$$

The apoptotic index was calculated from TUNEL-stained slides by determining the number of apoptotic nuclei per microscopic field at three central locations and three peripheral locations of the retina. The images were captured 
with a 40-fold objective magnification. Histopathological and morphometrical evaluations were performed by a toxicological pathologist certified by the Japanese Society of Toxicologic Pathology and the International Academy of Toxicologic Pathology (K. Y.) and an ophthalmologist certified by the Japanese Ophthalmological Society (M. K.), according to the previously defined histopathological terminology and diagnostic criteria ${ }^{(18,19)}$.

\section{Terminal deoxynucleotidyl transferase-mediated dUTP digoxigenin nick-end labelling staining}

Formalin-fixed retinal sections obtained from the rats (basal, 0.5 and $2.0 \% \mathrm{AA}$ ) that were killed $24 \mathrm{~h}$ after the MNU treatment were used for cell death analysis. Cell death was evaluated by TUNEL using an in situ apoptosis detection kit (ApopTag ${ }^{\circledR}$; Millipore) ${ }^{(19,23)}$.

\section{Statistical analysis}

All discrete values, expressed as means with their standard errors, were analysed using the two-tailed independent Student's $t$ test for unpaired samples after confirming the homogeneity of variances (Excel 2007; Microsoft). The results presented include comparisons between the basal diet-fed rats and rats fed the different doses of the AA-supplemented diet in both MNU-treated and vehicle-treated groups. $P$ values $<0.05$ were considered to show statistical significance.

\section{Results}

\section{General remarks}

No deaths occurred, and no clinical signs or symptoms were evident in any of the pups or dams during the experimental period. The AA diets did not influence body-weight gain (the growth rate) in pups or cause weight changes in dams with or without the MNU treatment; however, the growth rate in MNU-treated pups tended to be lower than that in vehicle-treated pups (data not shown).

\section{Estimated intake of arachidonic acid}

In the pregnancy and lactation periods, the AA intake of dams was 4.7 and $9.4 \mathrm{mg} / \mathrm{kg}$ per $\mathrm{d}$ in the basal diet group; 77.7 and $242.6 \mathrm{mg} / \mathrm{kg}$ per $\mathrm{d}$ in the $0.1 \%$ AA group; 261.8 and $874.0 \mathrm{mg} / \mathrm{kg}$ per $\mathrm{d}$ in the $0.5 \%$ AA group; and 1075.1 and 3058.5 $\mathrm{mg} / \mathrm{kg}$ per $\mathrm{d}$ in the $2.0 \%$ AA group, respectively.

\section{Morphological and morphometric analysis}

Retinal histology showed no abnormal changes in the central and peripheral retina in vehicle-treated rats that consumed any of the four diets; the retinas contained more than ten layers of photoreceptor nuclei in the central retina (Fig. 2(a)) and more than eight layers of cells in the peripheral retina (data not shown). At $7 \mathrm{~d}$ after a single MNU injection, the outer nuclear layer and the photoreceptor layer in the central retina disappeared or were reduced to a few rows of photoreceptor cell nuclei in rats fed a basal diet or $0 \cdot 1 \%$ AA diet (Fig. 2(b)). The remaining photoreceptor nuclei were densely stained and contained clumped chromatin. In contrast, the 0.5 and $2.0 \%$ AA diets rescued retinal damage. A waving array of retina was seen due to the focal disappearance of photoreceptor nuclei in the $0.5 \%$ AA diet group, while the retina showed almost normal histology in the $2.0 \%$ AA diet group. To further evaluate the effects of the AA diet on retinal thickness at the central retina, the photoreceptor cell ratio was calculated. MNU-treated rats that received the 0.5 and $2.0 \%$ AA diets had a statistically significant increase in their photoreceptor ratio compared with basal diet-fed rats (basal diet, 19.4\%;

(a)

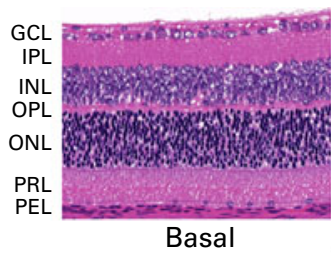

(b)

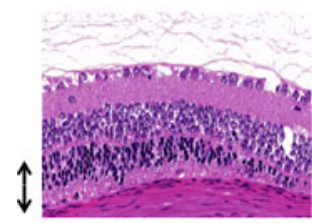

Basal

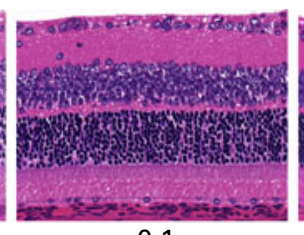

$0 \cdot 1$

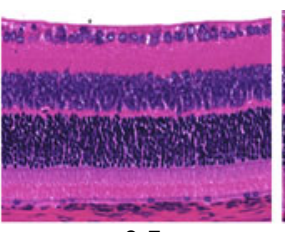

0.5

AA (\%)

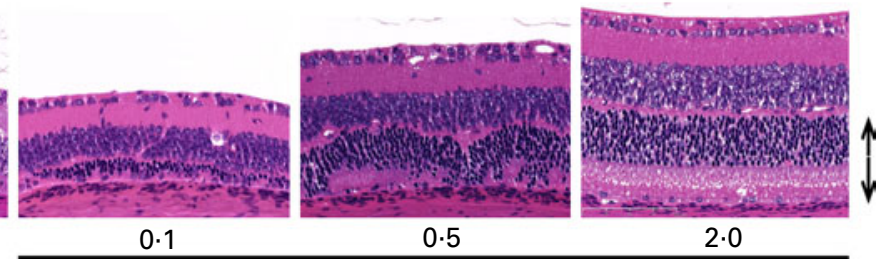

AA (\%)

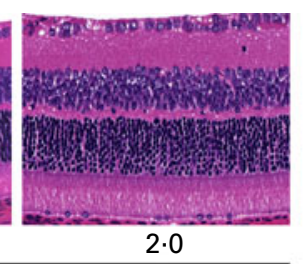

(2)

Fig. 2. Histology of the central retina in rats treated with (a) vehicle only or (b) $50 \mathrm{mg} / \mathrm{kg}$ of $N$-methyl- $N$-nitrosourea (MNU). Rats were fed the basal, $0.1,0.5$ and $2.0 \%$ arachidonic acid (AA) diets. At $7 \mathrm{~d}$ after a single intraperitoneal injection of MNU, the outer nuclear layer and the photoreceptor layer disappeared in rats fed the basal diet and the $0.1 \%$ AA diet (arrows). In contrast, the 0.5 and $2.0 \%$ AA diets prevented the retinal damage (arrows). GCL, ganglion cell layer; IPL, inner plexiform layer; INL, inner nuclear layer; OPL, outer plexiform layer; ONL, outer nuclear layer; PRL, photoreceptor cell layer; PEL, pigment epithelial cell layer. Haematoxylin and eosin staining, $200 \times$. 
$0.1 \%$ AA diet, $32 \cdot 0 \% ; 0.5 \%$ AA diet, $46.7 \% ; 2 \cdot 0 \%$ AA diet, $43.6 \%$; Fig. 3(a)). MNU-induced retinal degeneration initiates from the central retina and progresses to the peripheral retina ${ }^{(17)}$; thus, the peripheral retina in MNU-treated rats appeared normal in all the four diet groups. The morphometrical evaluation of the peripheral retina in MNU-treated rats showed that the photoreceptor ratio did not change in any of the diet groups. MNU-treated rats that received the 0.5 and $2.0 \%$ AA diets had a statistically significant decrease in the retinal damage ratio compared with basal diet-fed rats (basal diet, 39.5\%; 0.1\% AA diet, 21.2\%; 0.5\% AA diet, $2 \cdot 7 \% ; 2 \cdot 0 \%$ AA diet, $6 \cdot 1 \%$; Fig. 3(b)).

\section{Apoptotic index}

At $24 \mathrm{~h}$ after the $50 \mathrm{mg} \mathrm{MNU} / \mathrm{kg}$ treatment, TUNEL signals in the central retina of basal diet-fed rats appeared selectively in the photoreceptor cell nuclei; many pyknotic photoreceptor cell nuclei were TUNEL-positive without obvious nuclear destruction (Fig. 4(a)). TUNEL signals were detected much

(a)

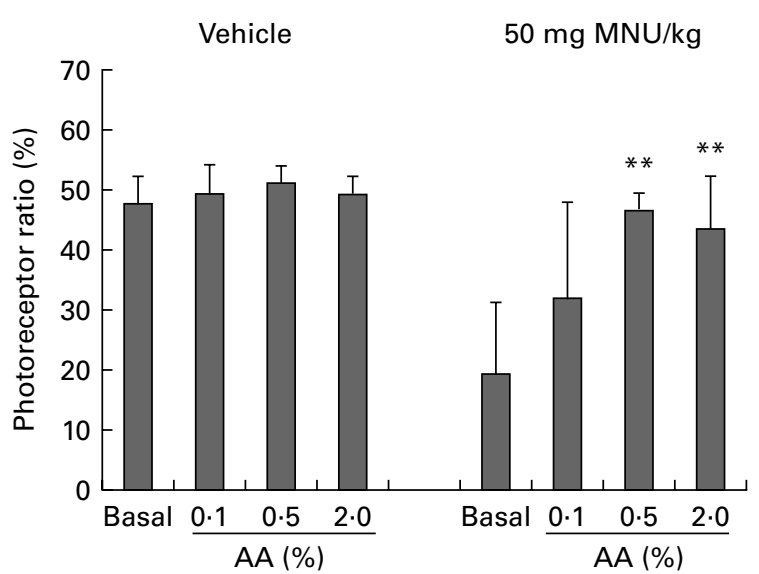

less frequently in the photoreceptor cell nuclei of the 0.5 and $2.0 \%$ AA groups. The apoptotic indices in the central retina of MNU-treated rats that received the 0.5 and $2.0 \% \mathrm{AA}$ diets were significantly less (21.9 and 14.9 , respectively) than the apoptotic index of MNU-treated rats that received the basal diet (37.6) (Fig. 4(b)). In the peripheral retina, the apoptotic indices of MNU-treated rats that received the 0.5 and $2.0 \%$ AA diets were comparable with that of MNU-treated rats that received the basal diet.

\section{Serum and retina fatty acid composition in N-methyl-N- nitrosourea-untreated rats}

In the serum of vehicle-treated rats that were fed the basal, $0 \cdot 1$, 0.5 and $2.0 \%$ AA diets, the AA compositions were 20.03, 22.66, 33.37 and $40.48 \mathrm{~mol} \%$ of total fatty acids, and the AA levels were $0.37,0.42,0.62$ and $0.74 \mu \mathrm{g} / \mu \mathrm{l}$, respectively. The AA compositions and levels in 0.5 and $2.0 \% \mathrm{AA}$ diet-fed rats were significantly increased compared with those in rats fed a basal diet (Fig. 5). The serum AA:DHA ratio was significantly

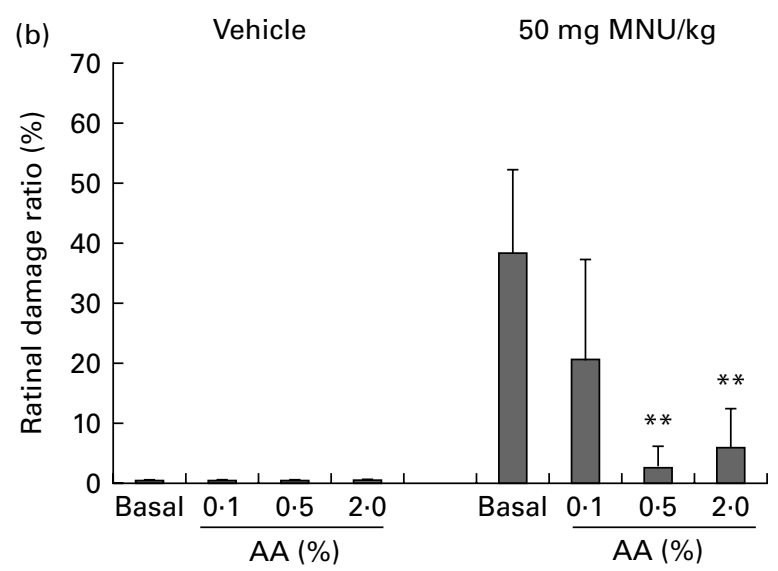

Fig. 3. Photoreceptor cell ratio in (a) the central retina and peripheral retina $7 \mathrm{~d}$ after a single intraperitoneal injection of $N$-methyl- $N$-nitrosourea (MNU) into rats fed the basal, $0.1,0.5$ and $2.0 \%$ arachidonic acid (AA) diets. Rats treated with $50 \mathrm{mg} \mathrm{MNU} / \mathrm{kg}$ that received the 0.5 or $2.0 \%$ AA diet had a statistically significant increase in their photoreceptor ratio at the central retina, compared with basal diet-fed rats. The index was calculated as: (outer retinal thickness/total retinal thickness) $\times 100$. (b) Retinal damage ratio in MNU-treated rats fed the basal, $0.1,0.5$ and $2.0 \%$ AA diets. Rats fed the 0.5 or $2.0 \%$ AA diet had a statistically significant decrease in the retinal damage ratio compared with basal diet-fed rats. The index was evaluated as: (length of retina composed of less than four photoreceptor cells/whole retinal length) $\times 100$. Values are means of four to eight rats in each treatment group, with their standard errors represented by vertical bars. ${ }^{* *}$ Mean values were significantly different from those of the vehicle-treated group $(P<0.01)$. 
(a)

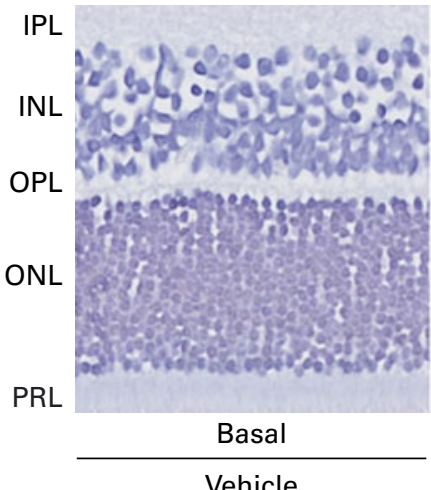

Vehicle

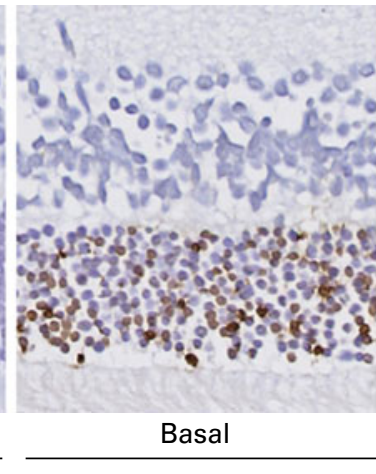

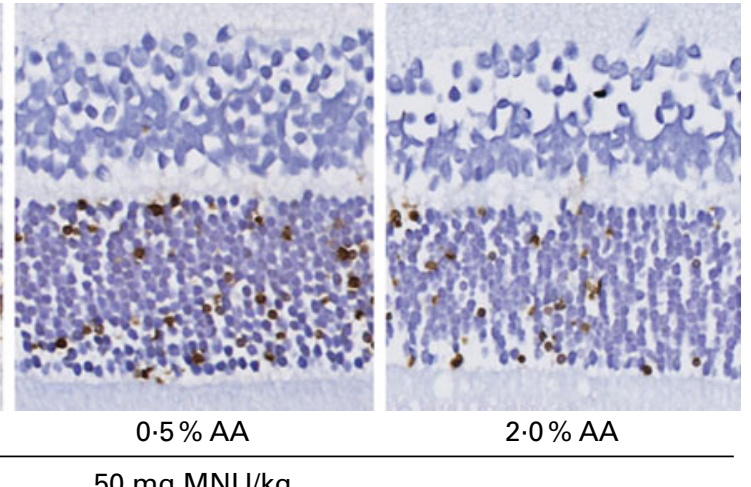

$50 \mathrm{mg} \mathrm{MNU/kg}$ (b)

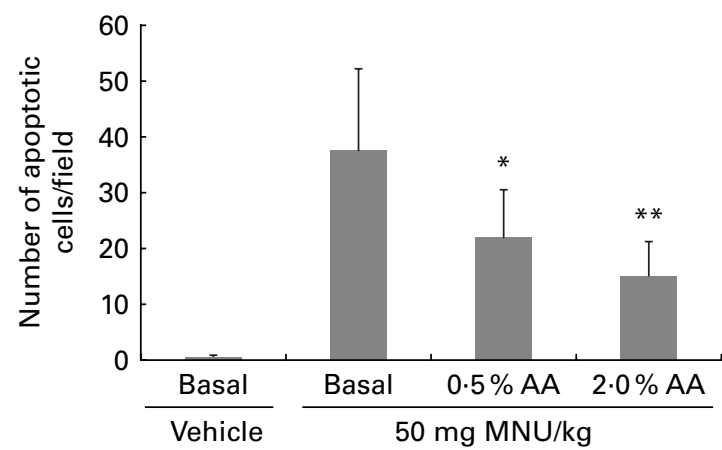

Central retina ${ }^{\star *} P<0.01$.

Fig. 4. Changes in the expression of terminal deoxynucleotidyl transferase-mediated dUTP digoxigenin nick-end labelling (TUNEL)-positive photoreceptor cells in the outer nuclear layer of the central retina. (a) At $24 \mathrm{~h}$ after a single intraperitoneal injection of $N$-methyl- $N$-nitrosourea (MNU) into rats fed the basal, 0.5 or $2.0 \%$ arachidonic acid (AA) diet, the signals are seen in many photoreceptor cell nuclei of rats fed the basal diet. However, the signals are seen in only some photoreceptor cell nuclei of rats fed the $2.0 \%$ AA diet. IPL, inner plexiform layer; INL, inner nuclear layer; OPL, outer plexiform layer; ONL, outer nuclear layer; PRL, photoreceptor cell layer. TUNEL staining, $400 \times$. The apoptotic index was evaluated in (b) the central retina and peripheral retina, respectively. Values are means of five rats, with their standard errors represented by vertical bars. Mean values were significantly different from those of the vehicle-treated group: ${ }^{\star} P<0.05$,

increased in 0.5 and $2.0 \%$ AA diet-fed rats compared with that in rats fed the basal diet; the AA:DHA ratio was 3.92, 4.20., 8.86 and 18.00 in basal, $0.1,0.5$ and $2.0 \%$ AA diet-fed rats, respectively. In contrast, in the retinas of vehicle-treated rats that were fed the basal, $0 \cdot 1,0.5$ and $2 \cdot 0 \%$ AA diets, the AA compositions were $6.61,7.58,7.36$ and $8.85 \mathrm{~mol} \%$ of total fatty acid, and the AA levels were $1.60,1.70,1.78$ and $2.18 \mu \mathrm{g} / \mathrm{mg}$, respectively. The $\mathrm{AA}$ compositions and levels in $2.0 \% \mathrm{AA}$ diet-fed rats were significantly increased compared with those in rats fed the basal diet (Fig. 5). The AA:DHA ratio was significantly increased in rats fed the $2.0 \%$ AA diet compared with that in rats fed a basal diet; the values were 0.46 , $0.48,0.54$ and 0.63 in the basal, $0.1 \%, 0.5 \%$ and $2.0 \%$ AA diet groups, respectively. In the basal, $0 \cdot 1 \%, 0.5 \%$ and $2 \cdot 0 \%$ AA diet groups, the total fatty acid levels were $1 \cdot 9,1 \cdot 7,2 \cdot 1$ and $1 \cdot 8 \mu \mathrm{g} / \mu \mathrm{l}$ in serum and $25 \cdot 0,23 \cdot 1,25 \cdot 1$ and $26 \cdot 3 \mu \mathrm{g} / \mathrm{mg}$ in the retina, respectively (NS).

\section{Discussion}

The present study examines the protective effects of dietary AA supplementation during the gestational, lactational and post-weaning periods against MNU-induced retinal degeneration in young rats. Morphological and morphometric evaluations indicate that a diet containing 0.5 or $2.0 \% \mathrm{AA}$ can counteract the effects of MNU and protect against MNU-induced retinotoxicity. Cell loss via apoptosis occurs in the course of human RP, as well as in mutant mouse models ${ }^{(9,14,15)}$. Apoptosis has been considered as a final common pathway of photoreceptor cell death. In the present study, 0.5 and $2.0 \%$ AA supplementation during prenatal development and early life inhibited the occurrence of apoptotic photoreceptor cell death $24 \mathrm{~h}$ after a single intraperitoneal injection of MNU.

AA and DHA are long-chain PUFA found in breast milk. Recently, it has been recommended that these PUFA be added to infant formula $(0 \cdot 2-0 \cdot 5 \% \text { of total fatty acids })^{(24)}$. AA and DHA play a pivotal role in neurodevelopment and the response to neural injury in the neonatal stage ${ }^{(5)}$, and they are the major structural long-chain PUFA in retinal photoreceptor outer segments ${ }^{(25)}$. The amounts of fatty acid in retinal tissue are modifiable by the composition of dietary fatty acid intake ${ }^{(20,26)}$. The retinal levels of AA and/or DHA are lower in rats with rhodopsin mutation ${ }^{(27)}$ and in $r d$ mice $^{(28)}$ with inherited retinal degeneration, and the plasma levels are lower in patients with human RP (Usher syndrome) ${ }^{(29)}$. In the present study, the serum and retinal levels of AA and the ratio of AA:DHA increased in vehicle-treated rats fed the 0.5 or $2.0 \%$ AA diet compared with rats fed a basal diet (containing $<0.01 \%(\mathrm{w} / \mathrm{w})$ AA). Retinal AA levels in the 
(a)
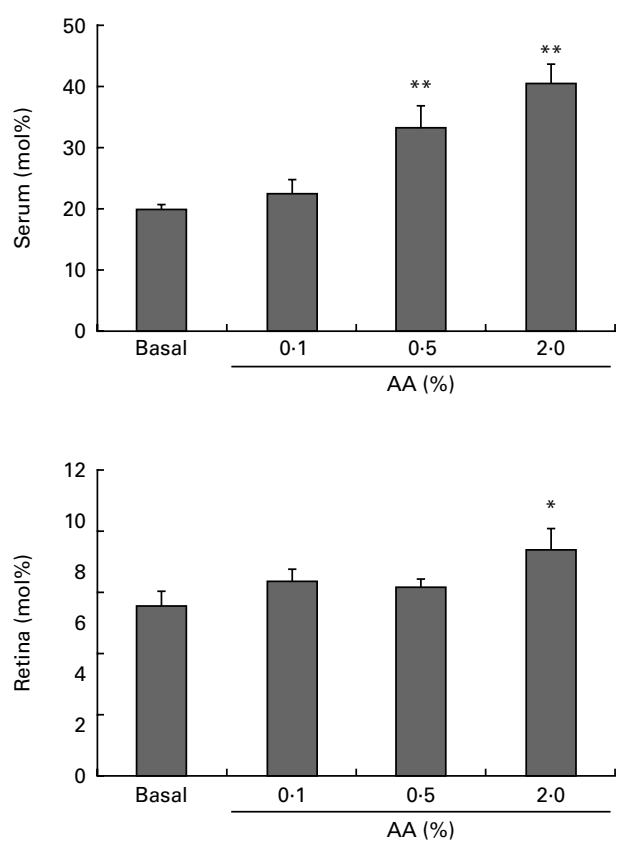

(b)
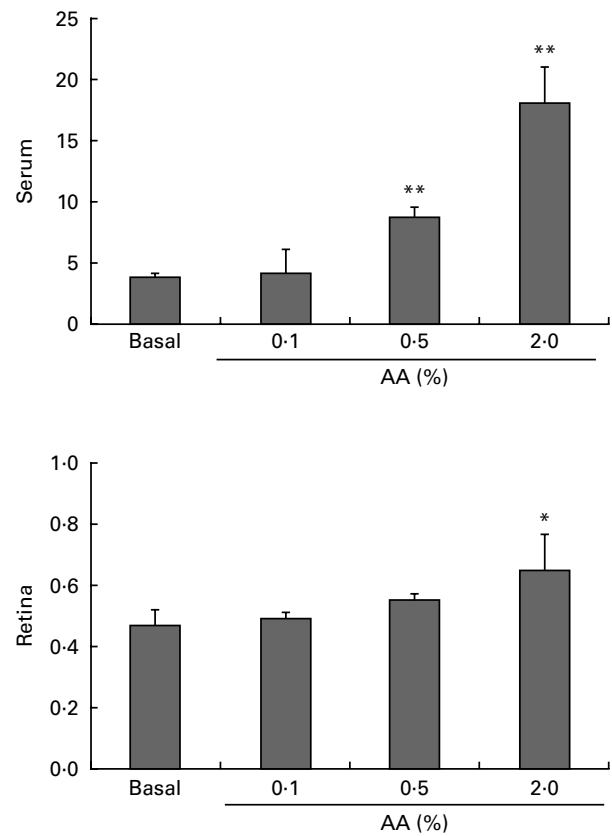

Fig. 5. Fatty acid composition of the retinas and serum of vehicle-treated rats. In the serum of rats fed a 0.5 or $2.0 \%$ arachidonic acid (AA) diet, (a) the AA composition of total fatty acids and (b) the AA:DHA ratio increased compared with those of basal diet-fed rats. In the retinas of rats fed a $2.0 \%$ (AA) diet, (a) the AA composition and (b) the AA:DHA ratio increased compared with those of basal diet-fed rats. Values are means of four to eight rats in each treatment group, with their standard errors represented by vertical bars. Mean values were significantly different from those of the basal diet-fed group: ${ }^{\star} P<0.05$, ${ }^{\star \star} P<0.01$.

MNU-treated groups were not analysed, because different degrees of rod outer segment damage affected by an AA-rich diet may alter the levels and create misleading results. However, AA administered during early developmental stages may protect against MNU-induced photoreceptor cell degeneration in the rat retina.

The visual and cognitive development of humans is supported through 39 months of age by the consumption of infant formula supplemented with AA ( $0 \cdot 43 \%$ of total fatty acids) and DHA (0.12\% of total fatty acids) until the age of 1 year $^{(30)}$. Visual maturation progression (a higher level of visual-evoked potential acuity) occurred in infants who received formulas supplemented with AA $(0.72 \%$ of total fatty acids) and DHA (0.36\% of total fatty acids) until 1 year of age ${ }^{(31)}$. Consensus recommendations by the World Association of Perinatal Medicine and other societies specify that infant formula should contain DHA in the range of $0 \cdot 2-0.5 \%$ of total fatty acids and AA at a level that is at least equal to that of $\mathrm{DHA}^{(24)}$. Dietary DHA is effective in rescuing MNU-induced retinal degeneration in adult rats ${ }^{(32,33)}$. High DHA composition during the induction/signalling phase and/or the effector phase of retinal cell apoptosis can delay the onset of cell death and counteract the progression of retinal degeneration in rodents ${ }^{(34)}$. In the present dietary dose regimen, the percentage of AA in total fatty acids and the AA:DHA ratio were extremely high (Table 1) compared with the recommended ratio in humans. In studies of retinal neurons in culture, DHA supplementation decreased photoreceptor cell death, whereas palmitic acid, oleic acid and AA were unable to decrease photoreceptor cell degeneration $^{(34-36)}$. However, in cultured brain neurons, AA shows biphasic actions at a narrow concentration range with the induction of cell death, on the one hand, and the promotion of cell survival and the enhancement of neurite extension, on the other hand ${ }^{(37)}$. The neurotoxic action is mediated by free radicals generated by AA metabolism, whereas the neurotrophic actions are exerted by AA itself ${ }^{(37,38)}$. This evidence suggests that dietary AA supplementation may be beneficial as a potential means to delay the onset and/or progression of neural disease by the inhibition of neuronal cell death at narrow windows of susceptibility (in the developmental phase) for photoreceptor rescue. In the present study, there were no abnormal changes in the retinas or brains (data not shown) of vehicle-treated rats that were fed any of the AA-supplemented diets $(0.1,0.5$ or $2.0 \%)$ at the developmental stage. The 0.5 and $2.0 \%$ AA diets or the $2.0 \%$ AA diet significantly elevated serum and retinal AA levels as well as AA:DHA levels, respectively, and rescued photoreceptors from apoptosis.

In human subjects, AA supplementation by healthy adults appears to confer no toxicity or significant safety risk; daily dosages of $1500 \mathrm{mg}$ for $50 \mathrm{~d}$ in the USA and $838 \mathrm{mg}$ for $14 \mathrm{~d}$ in Japan have been well tolerated in clinical studies with no significant side effects ${ }^{(39,40)}$. The recommended intake of AA in Japan is $24 \mathrm{mg} / \mathrm{kg}$ per $\mathrm{d}$ in adult human subjects ${ }^{(41)}$. Thus, in the basal, $0 \cdot 1,0.5$ and $2.0 \%$ AA diets, the AA dose in the pregnancy period was $0 \cdot 2,3 \cdot 2,10.9$ and 44.8 times higher than the recommended human dose, and the AA dose in the lactation period was $0 \cdot 4,10 \cdot 1,36 \cdot 4$ and $127 \cdot 4$ times higher than the recommended human dose, respectively. The average dietary AA intake was recently estimated to be $57 \mathrm{mg} / \mathrm{d}$ in healthy Canadian children between 4 and 
7 years of age ${ }^{(42)}$. The American College of Toxicology reviewed the safety and pharmacological assessments of AA, and found that exogenous AA appears to help prevent teratogenic effects in animal models and that a daily oral dose of $3000 \mathrm{mg} \mathrm{AA} / \mathrm{kg}$ for $27 \mathrm{~d}$ shows no side effects in rats ${ }^{(43)}$. Therefore, a higher dose of AA is tolerable and is needed for photoreceptor rescue as well as anti-teratogenic action in animals. Recently, female but not male newborn mice from $\mathrm{C} 57 \mathrm{BL} / 6$ dams fed $\mathrm{AA}(+) / \mathrm{DHA}(+)$ diets had a higher incidence of eye abnormalities (microphthalmia and corneal opacity) ${ }^{(44)}$. However, a high incidence of eye abnormalities exists in both female and male $\mathrm{C} 57 \mathrm{BL} / 6$ mice in historical control data ${ }^{(45)}$. Therefore, further study is needed to determine whether AA exposure is the cause of sex-dependent eye abnormalities in $\mathrm{C} 57 \mathrm{BL} / 6$ mice.

In conclusion, AA-rich diets (0.5 and $2.0 \% \mathrm{AA})$ during the gestational, lactational and post-weaning periods prevent MNU-induced retinal degeneration in young rats via the inhibition of photoreceptor apoptosis. An AA-rich diet induces increased levels of retinal AA, probably followed by the promotion of retinal development and maturity. Several factors including AA itself in the retina may inhibit photoreceptor cell death via the apoptosis cascade in MNU-induced retinal degeneration. The induction/signalling phase and/or the effector phase of retinal cell apoptosis may delay the onset of cell death and counteract the progression of retinal degeneration in rodents fed an AA-rich diet, similarly to high DHA composition ${ }^{(32-34)}$. Moreover, the detailed analysis of AA metabolites (cyclo-oxygenase and PG) in the retina of rats fed AA-rich diets may be necessary to understand the relationship to the rescue from MNU-induced retinal degeneration. The fatty acid composition of the prenatal and postnatal diet may affect the response to photoreceptor injury in human RP. Further studies of the photoreceptor apoptosis cascade are necessary to understand the detailed mechanisms by which AA prevents MNU-induced retinal degeneration.

\section{Acknowledgements}

This study was supported in part by a Grant-in Aid for Scientific Research (C) from the Japan Society for the Promotion of Science (22591954) and Health and Labour Sciences Research Grants (H22-Shokuhin-Ippan-002). We thank Ms T. Akamatsu for her excellent technical assistance and Ms A. Shudo for manuscript preparation. The authors' responsibilities were as follows: K. Y., T. S. and N. U. designed the study and provided technical expertise; K. Y. and M. K. conducted the study and histopathological analysis; A. H., N. O. and T. M. conducted and/or estimated the fatty acid analysis; K. Y., H. T. and A. T. analysed the data and wrote the manuscript. All authors read and approved the final manuscript. The authors declare that they have no competing interests.

\section{References}

1. Arterburn LM, Hall EB \& Oken H (2006) Distribution, interconversion, and dose response of $n-3$ fatty acids in humans. Am J Clin Nutr 83, 1467S-1476S.
2. Semba RD (2007) Essential fatty acids and visual development in infants. In Handbook of Nutrition and Ophthalmology, pp. 415-441 [RD Semba, editor]. New Jersey: Humana Press.

3. Le HD, Meisel JA, de Meijer VE, et al. (2009) The essentiality of arachidonic acid and docosahexaenoic acid. Prostaglandins Leukot Essent Fatty Acids 81, 165-170.

4. Davis-Bruno K \& Tassinari MS (2011) Essential fatty acid supplementation of DHA and ARA and effects on neurodevelopment across animal species: a review of the literature. Birth Defects Res (Part B) 92, 240-250.

5. Saste MD, Carver JD, Stockard JE, et al. (1998) Maternal diet fatty acid composition affects neurodevelopment in rat pups. J Nutr 128, 740-743.

6. Maekawa M, Takashima N, Matsumata M, et al. (2009) Arachidonic acid drives postnatal neurogenesis and elicits a beneficial effect on prepulse inhibition, a biological trait of psychiatric illnesses. PLoS One 4, e5085.

7. Uauy R, Hoffman DR, Peirano P, et al. (2001) Essential fatty acids in visual and brain development. Lipids 36, 885-895.

8. Age-Related Eye Disease Study Research Group (2007) The relationship of dietary lipid intake and age-related macular degeneration in a case-control study. Arch Ophthalmol 125, 671-679.

9. Hartong DT, Berson EL \& Dryja TP (2006) Retinitis pigmentosa. Lancet 368, 1795-1809.

10. Shintani K, Shechtman DL \& Gurwood AS (2009) Review and update: current treatment trends for patients with retinitis pigmentosa. Optometry 80, 384-401.

11. RetNet ${ }^{\mathrm{TM}}$. Retinal Information Network. American Red Cross. https://sph.uth.tmc.edu/Retnet/

12. Ferrari S, Di Iorio E, Barbaro V, et al. (2011) Retinitis pigmentosa: genes and disease mechanisms. Curr Genomics 12, $238-249$.

13. Williams DS (2008) Usher syndrome; animal models, retinal function of Usher proteins, and prospects for gene therapy. Vision Res 48, 433-441.

14. Rivas MA \& Vecino E (2009) Animal models and different therapies for treatment of retinitis pigmentosa. Histol Histopathol 24, 1295-1322.

15. Sancho-Pelluz J, Arango-Gonzalez B, Kustermann S, et al. (2008) Photoreceptor cell death mechanisms in inherited retinal degeneration. Mol Neurobiol 38, 253-269.

16. Tsubura A, Yoshizawa K, Kuwata M, et al. (2010) Animal models for retinitis pigmentosa induced by MNU; disease progression, mechanisms and therapeutic trials. Histol Histopathol 25, 933-944.

17. Yoshizawa K \& Tsubura A (2005) Characteristics of $N$-methyl$\mathrm{N}$-nitrosourea-induced retinal degeneration in animals and application for the therapy of human retinitis pigmentosa. Nippon Ganka Gakkai Zasshi 109, 327-337 (abstract in English; text in Japanese).

18. Yoshizawa K, Nambu H, Yang J, et al. (1999) Mechanisms of photoreceptor cell apoptosis induced by $N$-methyl- $N$-nitrosourea in Sprague-Dawley rats. Lab Invest 79, 1359-1367.

19. Yoshizawa K, Kuwata M, Kawanaka A, et al. (2009) $N$-Methyl- $N$-nitrosourea induced retinal degeneration in mice is independent of the $p 53$ gene. Mol Vis 15, 2919-2925.

20. Hussein N, Fedorova I, Moriguchi T, et al. (2009) Artificial rearing of infant mice leads to $n-3$ fatty acid deficiency in cardiac, neural and peripheral tissues. Lipids 44, 685-702.

21. Harauma A \& Moriguchi T (2011) Dietary $n-3$ fatty acid deficiency in mice enhances anxiety induced by chronic mild stress. Lipids 46, 409-416. 
22. Masood A, Stark KD \& Salem N Jr (2005) A simplified and efficient method for the analysis of fatty acid methyl esters suitable for large clinical studies. J Lipid Res 46, 2299-2305.

23. Yoshizawa K, Sasaki T, Uehara N, et al. (2011) $N$-Ethyl$\mathrm{N}$-nitrosourea induces retinal photoreceptor damage in adult rats. $J$ Toxicol Pathol 25, 27-35.

24. Hoffman DR, Boettcher JA \& Diersen-Schade DA (2009) Toward optimizing vision and cognition in term infants by dietary docosahexaenoic and arachidonic acid supplementation: a review of randomized controlled trials. Prostaglandins Leukot Essent Fatty Acids 81, 151-158.

25. Neuringer M (1993) The relationship of fatty acid composition to function in the retina and visual system. In Lipids, Learning, and the Brain: Fats in Infant Formulas, Report of the 103rd Ross Conference on Pediatric Research, pp. 134-163 [J Dobbing, editor]. Columbus, OH: Ross Laboratories.

26. Suh M, Wierzbicki AA, Lien EL, et al. (2000) Dietary $20: 4 n-6$ and 22:6n-3 modulates the profile of long- and verylong-chain fatty acids, rhodopsin content, and kinetics in developing photoreceptor cells. Pediatr Res 48, 524-530.

27. Anderson RE, Maude MB, McClellan M, et al. (2002) Low docosahexaenoic acid levels in rod outer segments of rats with $\mathrm{P} 23 \mathrm{H}$ and S334ter rhodopsin mutations. Mol Vis $\mathbf{8}$, 351-358.

28. Scott BL, Racz E, Lolley RN, et al. (1988) Developing rod photoreceptors from normal and mutant $r d$ mouse retinas: altered fatty acid composition early in development of the mutant. J Neurosci Res 20, 202-211.

29. Bazan NG, Scott BL, Reddy TS, et al. (1986) Decreased content of docosahexaenoate and arachidonate in plasma phospholipids in Usher's syndrome. Biochem Biophys Res Commun 141, 600-604.

30. Auestad N, Scott DT, Janowsky JS, et al. (2003) Visual, cognitive, and language assessments at 39 months: a follow-up study of children fed formulas containing long-chain polyunsaturated fatty acids to 1 year of age. Pediatrics $\mathbf{1 1 2}$, e177-e183.

31. Birch EE, Castaneda YS, Wheaton DH, et al. (2005) Visual maturation of term infants fed long-chain polyunsaturated fatty acid-supplemented or control formula for $12 \mathrm{mo} . \mathrm{Am} \mathrm{J}$ Clin Nutr 81, 871-879.

32. Moriguchi K, Yuri T, Yoshizawa K, et al. (2003) Dietary docosahexaenoic acid protects against $N$-methyl- $N$-nitrosoureainduced retinal degeneration in rats. Exp Eye Res 77, 167-173.
33. Tsubura A, Yuri T, Yoshizawa K, et al. (2009) Role of fatty acids in malignancy and visual impairment: epidemiological evidence and experimental studies. Histol Histopathol 24, 223-234.

34. Moriguchi K, Yoshizawa K, Shikata N, et al. (2004) Suppression of $N$-methyl- $N$-nitrosourea-induced photoreceptor apoptosis in rats by docosahexaenoic acid. Ophthalmic Res 36, 98-105.

35. Politi L, Rotstein N \& Carri N (2001) Effects of docosahexaenoic acid on retinal development: cellular and molecular aspects. Lipids 36, 927-935.

36. Rotstein NP, Aveldano MI, Barrantes FJ, et al. (1996) Docosahexaenoic acid is required for the survival of rat retinal photoreceptors in vitro. J Neurochem 66, 1851-1859.

37. Katsuki H \& Okuda S (1995) Arachidonic acid as a neurotoxic and neurotrophic substance. Prog Neurobiol 46, 607-636.

38. Kim HY, Akbar M \& Kim KY (2001) Inhibition of neuronal apoptosis by polyunsaturated fatty acid. J Mol Neurosci 16, 223-278.

39. Kusumoto A, Ishikura Y, Kawashima H, et al. (2007) Effects of arachidonate-enriched triacylglycerol supplementation on serum fatty acids and platelet aggregation in healthy male subjects with a fish diet. BrJ Nutr 98, 626-635.

40. Nelson GJ, Schmidt PC, Bartolini G, et al. (1997) The effect of dietary arachidonic acid on platelet function, platelet fatty acid composition, and blood coagulation in humans. Lipids 32, 421-425.

41. Suntory wellness website, Arabita. http://www.suntorykenko.com/supplement/main/433461 (in Japanese)

42. Lien VW \& Clandinin MT (2009) Dietary assessment of arachidonic acid and docosahexaenoic acid intake in 4-7 year-old children. J Am Coll Nutr 28, 7-15.

43. Anonymous (1993) Final report on the safety assessment of arachidonic acid. I Am Coll Toxicol 12, 481-559.

44. Maekawa M, Iwayama Y, Watanabe A, et al. (2010) Excessive ingestion of long-chain polyunsaturated fatty acids during developmental stage causes strain- and sex-dependent eye abnormalities in mice. Biochem Biophys Res Commun 402, 431-437.

45. Smith RS \& Sundberg JP (2002) Strain background disease characteristics. In Systematic Evaluation of the Mouse Eye: Anatomy, Pathology, and Biomethods, pp. 67-75 [RS Smith, editor]. Boca Raton, FL: CRC Press. 